\title{
MODELING THE UNIVERSE AS A QUANTUM WELL
}

\author{
${ }^{1}$ Michael Harney and ${ }^{2}$ Julie Seal \\ ${ }^{1}$ Johns Hopkins University, Baltimore, MD 21218, Maryland, USA \\ ${ }^{2}$ Brigham Young University, Provo, UT 84602, USA
}

Received 2014-02-05; Revised 2014-03-17; Accepted 2014-03-19

\begin{abstract}
By modeling the Universe as a quantum well for standing waves and solving Schrodinger's equation for a particle trapped in this well, the solutions for the allowable rest-energies of all particles in the Universe are obtained, from the mass of the photon up to the mass of the Universe. The quantum numbers that predict the allowable rest-energies of all particles are also found to quantize the distance over which gravity acts, ranging from the Planck length to the radius of the universe. The potential that is derived from the quantum well model is validated for gravitational, strong, weak and electromagnetic forces by confirming the mass of the vector particle for each force.
\end{abstract}

Keywords: Vector Particl, Electromagnetic Forces, Quantum Well

\section{INTRODUCTION}

It is believed that some form of quantization of the gravitational field exists and one approach to understanding this quantization is to map the field to a quantum well and solve for all of its associated nodes. If we assume these standing waves are DeBroglie waves correspond to the particles in our universe, then we can assign the resulting well potential for each series of quantum numbers to the rest energy of a particle. Dirac found with his Large Number Hypothesis (LNH) that there appears to be a relation between the ratio of magnitude between forces and the scale of the Universe. The quantum well model discussed in this study shows a similar relation and provides an explanation for Dirac's LNH. Based on the model discussed, the energy in a particular DeBroglie wave is equal to the rest-energy of a particle which may be localized by its probabilitydensity function. The effect of wave interaction within the well may be similar to Mach's principle where it can be shown that the rest-energy of any object is equivalent to the universal gravitational potential energy acting on that object (Harney, 2004).

We start by calculating the smallest mass that can occur in the two-dimensional plane where DeBroglie waves travel. This is done by solving Schrodinger's equation for the fundamental standing wavelength in a two-dimensional system with dimensions equal to twice the radius of the universe. In this process, we find all the allowable rest energies that the particle can have based on quantum numbers for $\mathrm{x}$ and $\mathrm{y}$ as follows (Krane, 1983) Equation 1:

$$
E=\left[\left(n_{x}\right)^{2}+\left(n_{y}\right)^{2}\right] \pi^{2} h^{2} /\left(2 m L^{2}\right)
$$

where, $\mathrm{n}_{\mathrm{x}}$ and $\mathrm{n}_{\mathrm{y}}$ are the quantum numbers, $\mathrm{m}$ is the mass of the particle, $\mathrm{L}$ is the length of the box or in this case, twice the radius of the universe (with the radius being equal to $1.9 \times 10^{26} \mathrm{~m}$ ) and $\mathrm{h}$ is Planck's constant of $6.62 \times 10^{-34}$ Joules-sec. By setting $\mathrm{n}_{\mathrm{x}}$ and $\mathrm{n}_{\mathrm{y}}$ equal to 1 and $\mathrm{L}$ equal to twice the radius of the universe, we obtain the ground state energy of a particle of fundamental matterwavelength inside the universe which we assume to be the photon, $\mathrm{m}_{\mathrm{p}}$. We then set the ground-state energy of the photon's matter-wave equal to its rest-energy Equation 2:

$$
2 \pi^{2} h^{2} /\left(2 m_{P} L^{2}\right)=m_{P} c^{2}
$$

By substituting $L=2 r$ where $r=1.9 \times 10^{26} \mathrm{~m}$ we find the mass of the photon to be Equation 3:

$$
m_{P}=\left[2 \pi^{2} h^{2} /\left(2(2 r)^{2} C^{2}\right)\right]^{1 / 2}=1.8 \times 10^{-68} \mathrm{~kg}
$$

This value for photon mass closely matches an estimate proposed by Vigier (1997) in his analysis of 
what was considered experimental error in the Michelson-Morley experiment. The Michelson-Morley experiment was designed to verify the existence of the medium that electromagnetic waves travel in (what we would view as a space-time fabric and what used to be called the ether). The experiment actually yielded a small ether drift of $8 \mathrm{~km} \mathrm{sec}^{-1}$ that was much smaller than the expected $320 \mathrm{~km} \mathrm{sec}^{-1}$ and this small drift was attributed to experimental error. An analysis of the data by Morley and others however, has shown that the "error" is periodic with respect to the rotation of the Earth and its periodicity is verified in other multiple experiments. Vigier has proposed a photon mass of $10^{-68} \mathrm{Kg}$ from the Einstein-DeBroglie relation that will offset the ether drift of $8 \mathrm{Km} \mathrm{sec}^{-1}$ and restore a relativistic outcome for the experiment, otherwise the ether drift indicates an absolute reference frame.

\subsection{Quantization of Mass and the Gravitational Field}

The underlying principle that is used for the above derivation of (2) and (3) is that rest-energy of the particle is equivalent to quantum well energy with the corresponding quantum numbers and it can be shown from Mach's principle that Universal Gravitational Potential Energy (UGPE) is equivalent to rest-mass energy (Harney, 2004), therefore quantum-well energy is then equivalent to UGPE, which allows us to quantize the Newtonian-gravitational potential as follows Equation 4:

$$
\left[\left(n_{x}\right)^{2}+\left(n_{y}\right)^{2}\right] \pi^{2} h^{2} /\left(2 m L^{2}\right)=m M_{u} G / r
$$

Where:

$\mathrm{r} \quad=$ Radius of known observable universe

$\mathrm{M}_{\mathrm{u}}=$ Mass of universe $\left(1.44 \times 10^{53} \mathrm{Kg}\right)$

$\mathrm{m}=$ Mass of object

Notice above that the quantization is more noticeable at larger potentials, on the order of galactic clusters, for instance, than it would be at a solar system level. This is what we would expect for a transition from Newtonian to Non-Newtonian gravitational model. As the quantum numbers get larger, the potential curve will obviously take on different shapes for the strong and weak regimes of particle interaction, similar to an elasticity plot for a given material. We will show later that this allows for a relationship similar to Hooke's law to be applied to space-time. It can be seen from (2) that this is a formula for the quantization of mass which can be defined as Equation 5: $m=\left[\left(n_{x}\right)^{2}+\left(n_{y}\right)^{2}\right] \pi^{2} h^{2} /\left(2(2 r)^{2} c^{2}\right)^{1 / 2}$

where, for the photon the quantum numbers are equal to 1 as we have just shown and for more massive, stable particles, the quantum numbers are larger. This equation assumes particles that do not decay. For the largest mass, the mass of the universe, we solve for the combination of $\left(\left(\mathrm{n}_{\mathrm{x}}\right)^{2}+\left(\mathrm{n}_{\mathrm{y}}\right)^{2}\right)$ by taking a ratio of two equations modeled after (5). By leaving the quantum numbers as variables in the numerator for the mass of the universe and setting them equal to 1 in the denominator for the mass of the photon (with all but quantum numbers cancelling on the left hand side) Equation 6:

$\left(\left(n_{x}\right)^{2}+\left(n_{y}\right)^{2}\right)=M_{u} / m_{P}=10^{121}$

Where:

$\mathrm{M}_{\mathrm{u}}=$ Mass of universe $\left(10^{53} \mathrm{~kg}\right)$

$\mathrm{m}_{\mathrm{p}}=$ Mass of photon $=10^{-68} \mathrm{~kg}$

And the effective quantum number $\left(n_{\text {eff }}\right.$, the quantum number of the same order of magnitude as either $\mathrm{n}_{\mathrm{x}}$ or $\mathrm{n}_{\mathrm{y}}$ ) is Equation 7:

$n_{\text {eff }}=\left[\left(\left(n_{x}\right)^{2}+\left(n_{y}\right)^{2}\right)\right]^{1 / 2}=10^{60}$

Which is similar to the results obtained from (Jordan, 1947; Shemi-Zadah, 2002).

The physical interpretation of these results is that rest-energy (and hence rest-mass, as c cancels from numerator and denominator in (6)) is quantized and can assume only certain values.

As a particle of higher mass than the photon will have a higher $n_{\text {eff }}$, which corresponds to a shorter DeBroglie wavelength, the standing waves in (1) will then have a wavelength given by Equation 8:

$\lambda=2 L / n$

where, $\mathrm{L}=2$ (radius of known observable universe). This last development corresponds directly with Heisenberg's Uncertainty principle Equation 9:

$\Delta E \Delta t=h$

where, $\Delta \mathrm{E}$ is found from (1) as Equation 10 and 11:

$\Delta E=\left(\Delta n_{\text {eff }}\right)^{2} \pi^{2} h^{2} /\left(2 m L^{2}\right)$ 
And:

$$
\Delta t=\Delta \lambda / C
$$

where, $\mathrm{c}=$ speed of light and $\Delta \lambda$ is found by differentiating (8) with respect to $n$ Equation 12:

$$
\Delta \lambda=-4 L /\left(\Delta n_{e f f}\right)^{2}
$$

Therefore, combining (10), (11) and (12) and recognizing $L=2$ r Equation 13 and 14:

$$
\begin{aligned}
& \Delta E \Delta t=\left[\left(\Delta n_{\text {eff }}\right)^{2} \pi^{2} h^{2} /\left(2 m L^{2}\right)\right] \\
& {\left[-4 L / c\left(\Delta n_{\text {eff }}\right)^{2}\right]=\pi^{2} h^{2} /(m r c)}
\end{aligned}
$$

Where:

$\mathrm{m}=$ Mass of photon $=1.8 \times 10^{-68} \mathrm{~kg}$

$\mathrm{r}=$ Radius of known observable universe $=1.9 \times 10^{26} \mathrm{~m}$

$\mathrm{c}=$ Speed of light

And:

$$
\Delta E \Delta t=\pi^{2} h^{2} /(m r c)=6 \times 10^{-34} J \mathrm{sec}=h
$$

This shows that an increase in energy (by increasing mass as in (10)) causes a decrease in $\Delta t$ (12), which makes $\Delta \mathrm{E} \Delta \mathrm{t}$ constant therefore, Heisenberg's uncertainty principle is derived from assuming a standing wave formula for all masses (with $\mathrm{n}=1$ corresponding to $\lambda=\mathrm{r}$ ) and applying Schrodinger's equation to calculate the energies in the standing waves. A photon has the lowest location resolution and the highest momentum resolution. The low-resolution of photon location (let's call it nonlocality) may explain photon entanglement, where photon's initially linked by metastable quantum states are capable of non-local communication.

As an object approaches the speed of light and it's relativistic energy approaches $\mathrm{M}_{\mathrm{u}} \mathrm{c}^{2}$, it's $\mathrm{n}_{\mathrm{eff}}$ approaches $10^{60}$ and it's matter-wavelength is reduced. This may be where the Planck length is most commonly noticed-as a limiting factor on $n_{\text {eff }}$ which in turn limits the velocity that an object can attain as it approaches the speed of light.

\subsection{Particle Interactions in the Quantized Field}

Now we examine how quantization affects the vector particles and their ranges for the four forces. The Planck length is defined as Equation 15:

$$
L_{P}=\left[(h G) /\left(2 \pi c^{3}\right)\right]^{1 / 2}=1.616 \times 10^{-35} \mathrm{~m}
$$

By knowing that the radius of the universe, $R_{u}$ is $1.9 \times 10^{26} \mathrm{~m}$ and knowing that the Planck length is the minimum distance the gravitational force can act over we find that Equation 16:

$$
\left(R_{u}\right) /\left(L_{P}\right)=n_{\text {eff }}=10^{61}
$$

Therefore, the maximum quantum number $\left(\mathrm{n}_{\mathrm{eff}}=\right.$ $10^{61}$ ) that corresponds to the ratio of the maximum to minimum rest-masses in the universe is also equal to the ratio of maximum to minimum radii that the gravitational force acts over. By incorporating (6) and (16) together we find Equation 17:

$$
G M_{u} / R_{u}^{2}=G m_{P} / L_{P}^{2}
$$

Or that the gravitational force from the mass of the universe acting on an object over the radius of the universe is equal to a photon's gravitational pull on the same object acting over the distance of the Planck length. This simply states that the electromagnetic force and its vector particle the photon has the same mass/range relationship on its minimum scale (Planck Length) as the gravitational force has over its maximum scale (mass of universe, radius of known observable universe). It is also interesting to note that the relationship in (17) applies to the strong nuclear force as well Equation 18:

$$
G M_{u} / R_{u}^{2}=G m_{p i(+) \text { meson }} / R_{\text {strong }}^{2}
$$

where, $\mathrm{m}_{\text {pi-meson }}=139.6 \mathrm{MeV}$ and $\mathrm{R}_{\text {strong }}$ is the distance the strong-nuclear force acts over (or maximum nuclear radius distance), which after solving (18) we find:

$$
R_{\text {Strong }}^{2}=7.88 \times 10^{-15} \mathrm{~m}
$$

Which is the maximum known nuclear radius and the limit of the range of the strong nuclear force. For the weak nuclear force, which has a generally accepted range of $10^{-18} \mathrm{~m}$ and using the constant evaluated in (17), the estimated mass of the vector particle (which is mostly likely the electron-neutrino) is $10^{-36} \mathrm{~kg}$ or 0.65 $\mathrm{eV} / \mathrm{c}^{2}$. In this respect, the strong and weak nuclear forces obey Newton's gravitational law at their maximum ranges as shown in (18). The gravitational force is therefore quantized at the level of the atom, with the strong and weak nuclear force exhibiting gravitational effects. 
Based on (2) where we assume that the rest-mass energy of a particle is equivalent to its quantum-well energy we formulate the conclusion that a particle can be viewed as a series of standing-wave ripples in the fabric of space-time, where the amplitude of the ripple is $\mathrm{mc}^{2}$ and this amplitude is also the vector axis of particle motion. The cartesian-coordinates that are normal to the particle's axis of motion correspond to the DeBroglie wave vectors specified by $\mathrm{n}_{\mathrm{x}}, \mathrm{n}_{\mathrm{y}}$ and $\mathrm{L}$ in (1). Also, as we have calculated a mass for the photon in (3) and assigned $n=1$ to it's DeBroglie wavelength while it travels at free-space velocity, we know that it's E and $\mathrm{H}$ vectors are normal to its direction of propogation and therefore the $\mathrm{E}$ and $\mathrm{H}$ fields are mapped into the same plane as it's DeBroglie waves, which explains why we used a 2-dimensional model for the quantum well. The amplitude of the photon's DeBroglie waves are found to be $\mathrm{m}_{\mathrm{p}} \mathrm{c}^{2}$ which is also equivalent to its gravitational potential as defined by (4), which allows for future derivations between the gravitational field and the electromagnetic fields.

\section{CONCLUSION}

The quantization of mass and gravitational fields can be interpreted by viewing these particles or fields as standing DeBroglie waves in a space-time fabric. The potential energy in this fabric is equivalent to the restenergy of particles and the potential energy of gravitational fields. The standing DeBroglie waves of a particle are projected in two-dimensions normal to the axis of motion of the particle. The $\mathrm{E}$ and $\mathrm{H}$ vectors of electromagnetic fields are mapped into the same plane as the DeBroglie waves, with interactions occurring as energy displacement inside the spacetime fabric. By applying Schrodinger's equation to this standing wave with the two-dimensional plane that it is in, the mass of particles can be interpreted from the quantized energy of the waves.

\section{REFERENCES}

Harney, M., 2004. Derivation of hubble's constant and the quantization of the gravitational field. J. Theor.

Krane, K.S., 1983. Modern Physics. 1st Edn., John Wiley and Sons, Toronto, ISBN-10: 0471079634, pp: 512.

Vigier, J.P., 1997. Relativistic interpretation (with nonzero photon mass) of the small ether drift velocity detected by michelson. Apeiron, 4: 71-76.

Jordan, P., 1947. Die Herkunft Der Sterne. 1st Edn., Wissenschaftliche Verlagsgesellschaft, Stuttgart, pp: 70.

Shemi-Zadah, V.E., 2002. Coincidence of large numbers, exact value of cosmological parameters and their analytical representation. Lebedev Physical Institute. 